\title{
A Compensatory Approach to Multiobjective Linear Transportation Problem with Fuzzy Cost Coefficients
}

\author{
Hale Gonce Kocken ${ }^{1}$ and Mehmet Ahlatcioglu ${ }^{2}$ \\ ${ }^{1}$ Department of Mathematical Engineering, Faculty of Chemistry-Metallurgy, Yildiz Technical University, \\ Davutpasa, 34220 Istanbul, Turkey \\ ${ }^{2}$ Department of Mathematics, Faculty of Arts and Science, Yildiz Technical University, \\ Davutpasa, 34220 Istanbul, Turkey
}

Correspondence should be addressed to Hale Gonce Kocken, halegk@gmail.com

Received 27 April 2011; Revised 16 July 2011; Accepted 9 August 2011

Academic Editor: J. J. Judice

Copyright (C) 2011 H. Gonce Kocken and M. Ahlatcioglu. This is an open access article distributed under the Creative Commons Attribution License, which permits unrestricted use, distribution, and reproduction in any medium, provided the original work is properly cited.

This paper deals with the Multiobjective Linear Transportation Problem that has fuzzy cost coefficients. In the solution procedure, many objectives may conflict with each other; therefore decision-making process becomes complicated. And also due to the fuzziness in the costs, this problem has a nonlinear structure. In this paper, fuzziness in the objective functions is handled with a fuzzy programming technique in the sense of multiobjective approach. And then we present a compensatory approach to solve Multiobjective Linear Transportation Problem with fuzzy cost coefficients by using Werner's $\mu_{\text {and }}$ operator. Our approach generates compromise solutions which are both compensatory and Pareto optimal. A numerical example has been provided to illustrate the problem.

\section{Introduction}

The classical transportation problem (TP) is a special type of linear programming problem, and it has wide practical applications in manpower planning, personnel allocation, inventory control, production planning, and so forth. TP aims to find the best way to fulfill the demand of $n$ demand points using the capacities of $m$ supply points. In a single objective TP, the cost coefficients of the objective express commonly the transportation costs. But in real-life situations, it is required to take into account more than one objective to reflect the problem more realistically, and thus multiobjective transportation problem (MOTP) becomes more useful. These objectives can be quantity of goods delivered, unfulfilled demand, average delivery time of the commodities, reliability of transportation, accessibility to the users, and product deterioration. Also in practice, the parameters of MOTP (supply\&demand 
quantities and cost coefficients) are not always exactly known and stable. This imprecision may follow from the lack of exact information, changeable economic conditions, and so forth. A frequently used way of expressing the imprecision is to use the fuzzy numbers. It enables us to consider tolerances for the model parameters in a more natural and direct way. Therefore, MOTP with fuzzy parameters seems to be more realistic and reliable.

A lot of researches have been conducted on MOTP with fuzzy parameters. Hussein [1] dealt with the complete solutions of MOTP with possibilistic coefficients. Das et al. [2] focused on the solution procedure of the MOTP where all the parameters have been expressed as interval values by the decision maker. Ahlatcioglu et al. [3] proposed a model for solving the transportation problem whose supply and demand quantities are given as triangular fuzzy numbers bounded from below and above, respectively. Basing on extension principle, Liu and Kao [4] developed a procedure to derive the fuzzy objective value of the fuzzy transportation problem where the cost coefficients, supply and demand quantities are fuzzy numbers. Using signed distance ranking, defuzzification by signed distance, intervalvalued fuzzy sets, and statistical data, Chiang [5] gets the transportation problem in the fuzzy sense. Ammar and Youness [6] examined the solution of MOTP which has fuzzy cost, source and destination parameters. They introduced the concepts of fuzzy efficient and $\alpha$-parametric efficient solutions. Islam and Roy [7] dealt with a multiobjective entropy transportation problem with an additional delivery time constraint, and its transportation costs are generalized trapezoidal fuzzy numbers. Chanas and Kuchta [8] proposed a concept of the optimal solution of the transportation problem with fuzzy cost coefficients and an algorithm determining this solution. Pramanik and Roy [9] showed how the concept of Euclidean distance can be used for modeling MOTP with fuzzy parameters and solving them efficiently using priority-based fuzzy goal programming under a priority structure to arrive at the most satisfactory decision in the decision making environment, on the basis of the needs and desires of the decision making unit.

In this paper, we focus on the solution procedure of the multiobjective linear Transportation Problem (MOLTP) with fuzzy cost coefficients. We assume that the supply and demand quantities are precisely known. And the coefficients of the objectives are considered as trapezoidal fuzzy numbers. The fuzziness in the objectives is handled with a fuzzy programming technique in the sense of multiobjective approach [10]. And a compensatory approach is given by using Werner's $\mu_{\text {and }}$ operator.

This paper is organized as follows. Section 2 presents brief information about fuzzy numbers. Section 3 contains the MOLTP formulation with fuzzy cost coefficients and some basic definitions about multiobjective optimization. Section 4 introduces the compensatory fuzzy aggregation operators briefly. Section 5 explains our methodology using Werners' compensatory "fuzzy and" operator. Section 6 gives two illustrative numerical examples. Finally, Section 7 includes some results.

\section{Fuzzy Preliminaries}

In this paper, we assumed that the fuzzy cost coefficients are trapezoidal fuzzy numbers. In this section, brief information about the fuzzy numbers especially trapezoidal fuzzy numbers is presented. For more detailed information, the reader should check [11, 12].

Definition 2.1. A fuzzy number $\widetilde{M}$ is an upper semicontinuous normal and convex fuzzy subset of the real line $R$. 
Definition 2.2. A fuzzy number $\widetilde{M}=\left(m_{1}, m_{2}, m_{3}, m_{4}\right)$ is said to be a trapezoidal fuzzy number (TFN) if its membership function is given by

$$
\mu_{\widetilde{M}}(x)= \begin{cases}0, & x<m_{1} \\ \frac{x-m_{1}}{m_{2}-m_{1}}, & m_{1} \leq x<m_{2} \\ 1, & m_{2} \leq x \leq m_{3} \\ \frac{m_{4}-x}{m_{4}-m_{3}}, & m_{3}<x \leq m_{4} \\ 0, & x>m_{4}\end{cases}
$$

where $m_{1}, m_{2}, m_{3}, m_{4} \in R$ and $m_{1} \leq m_{2} \leq m_{3} \leq m_{4}$. The figure of the fuzzy number $\widetilde{M}$ is given in Figure 1. It should be noted that a triangular fuzzy number is a special case of a TFN with $m_{2}=m_{3}$. In the literature, a TFN can also be represented with the ordered quadruplets $\left(m_{2}, m_{3}, m_{2}-m_{1}, m_{4}-m_{3}\right)$. Here $m_{2}-m_{1}$ is called as left spread of $\widetilde{M}$, where $m_{4}-m_{3}$ is right spread. In this paper, we use $\widetilde{M}=\left(m_{1}, m_{2}, m_{3}, m_{4}\right)$ fuzzy number notation and called these ordered elements as characteristic points of $\widetilde{M}$. follows.

Some algebraic operations on TFNs that will be used in this paper are defined as Let $\tilde{a}=\left(a_{1}, a_{2}, a_{3}, a_{4}\right)$ and $\tilde{b}=\left(b_{1}, b_{2}, b_{3}, b_{4}\right)$ be TFNs.

(i) Addition:

$$
\tilde{a}+\tilde{b}=\left(a_{1}+b_{1}, a_{2}+b_{2}, a_{3}+b_{3}, a_{4}+b_{4}\right)
$$

(ii) Multiplication with a Positive Crisp Number $(k>0)$ :

$$
k \cdot \tilde{a}=\left(k a_{1}, k a_{2}, k a_{3}, k a_{4}\right) .
$$

One convenient approach for solving the fuzzy linear programming problems is based on the concept of comparison of fuzzy numbers by use of ranking functions.

Definition 2.3. The set of elements that belong to the fuzzy number $\tilde{a}$ at least to the degree $\alpha$ is called the $\alpha$-level set: $\tilde{a}_{\alpha}=\left\{x \in X \mid \mu_{\tilde{a}}(x) \geq \alpha\right\}$. 


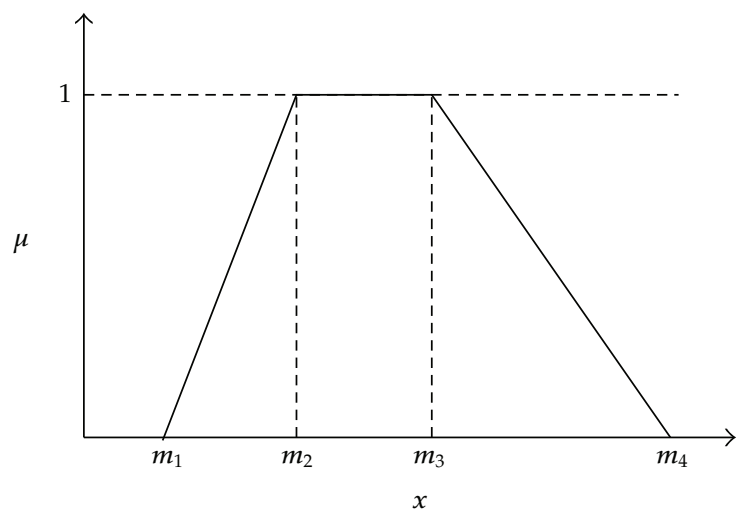

Figure 1: The membership function of $\widetilde{M}$.

Definition 2.4. Let $R: F(\mathbb{R}) \rightarrow \mathbb{R}$ be a ranking function which maps each fuzzy number into the real line, where a natural order exists. We denote the set of all trapezoidal fuzzy numbers by $F(R)$. The orders could be defined on $F(\mathbb{R})$ by

$$
\begin{aligned}
& \tilde{a} \geq_{R} \tilde{b} \quad \text { iff } R(\tilde{a}) \geq R(\tilde{b}), \\
& \tilde{a} \leq_{R} \tilde{b} \quad \text { iff } R(\tilde{a}) \leq R(\tilde{b}), \\
& \tilde{a}=\tilde{b} \quad \text { iff } R(\tilde{a})=R(\tilde{b}),
\end{aligned}
$$

where $\tilde{a}$ and $\tilde{b}$ are in $F(\mathbb{R})$. Also we write $\tilde{a} \leq_{R} \tilde{b}$ if and only if $\tilde{b} \geq_{R} \tilde{a}$. We restrict our attention to linear ranking functions, that is, a ranking function $R$ such that

$$
R(k \tilde{a}+\tilde{b})=k R(\tilde{a})+R(\tilde{b}) .
$$

In this paper, we used the linear ranking function which was first proposed by Yager [13]

$$
R(\tilde{a})=\frac{1}{2} \int_{0}^{1}\left(\inf \tilde{a}_{\curlywedge}+\sup \tilde{a}_{\curlywedge}\right) d \lambda
$$

which reduces to

$$
R(\tilde{a})=\frac{a_{1}+a_{2}+a_{3}+a_{4}}{4}
$$

Then, for TFNs $\tilde{a}$ and $\tilde{b}$, we have

$$
\tilde{a} \leq_{R} \tilde{b} \quad \text { iff } a_{1}+a_{2}+a_{3}+a_{4} \leq b_{1}+b_{2}+b_{3}+b_{4} .
$$




\section{The MOLTP with Fuzzy Cost Coefficients}

The MOLTP with fuzzy cost coefficients is formulated as follows:

$$
\begin{array}{ll}
\min \tilde{f}^{k}(\mathbf{x})=\sum_{i=1}^{m} \sum_{j=1}^{n} \widetilde{c}_{i j}^{k} x_{i j}, \quad k=1,2, \ldots, K, \\
\text { s.t.: } \quad \sum_{j=1}^{n} x_{i j}=a_{i}, \quad i=1,2, \ldots, m, \\
& \sum_{i=1}^{m} x_{i j}=b_{j}, \quad j=1,2, \ldots, n, \\
& x_{i j} \geq 0, \quad i=1,2, \ldots, m ; j=1,2, \ldots, n .
\end{array}
$$

$x_{i j}$ is decision variable which refers to product quantity that is transported from supply point $i$ to demand point $j . a_{1}, a_{2}, \ldots, a_{m}$ and $b_{1}, b_{2}, \ldots, b_{n}$ are $m$ supply and $n$ demand quantities, respectively. We note that $a_{i}(i=1,2, \ldots, m)$ and $b_{j}(j=1,2, \ldots, n)$ are crisp numbers. $K$ is the number of the objective functions of MOLTP. $\tilde{c}_{i j}^{k}$ is fuzzy unit transportation cost from supply point $i$ to demand point $j$ for the objective $k,(k=1,2, \ldots, K)$. For our fuzzy transportation problem, the coefficients of the objectives $\tilde{c}_{i j}^{k}$ are considered as trapezoidal fuzzy numbers:

$$
\tilde{c}_{i j}^{k}=\left(c_{i j}^{k 1}, c_{i j}^{k 2}, c_{i j}^{k 3}, c_{i j}^{k 4}\right)
$$

The membership function of the fuzzy number $\tilde{c}_{i j}^{k}$ and its figure are given in (3.3) and Figure 2, respectively,

$$
\mu_{\tilde{c}_{i j}^{k}}(x)= \begin{cases}0, & x<c_{i j}^{k 1}, \\ \frac{x-c_{i j 1}^{k 1}}{c_{i j}^{k 2}-c_{i j}^{k 1}}, & c_{i j}^{k 1} \leq x<c_{i j}^{k 2}, \\ 1, & c_{i j}^{k 2} \leq x \leq c_{i j}^{k 3}, \\ \frac{c_{i j}^{k 4}-x}{c_{i j}^{k 4}-c_{i j}^{k 3}}, & c_{i j}^{k 3}<x \leq c_{i j}^{k 4}, \\ 0, & x>c_{i j}^{k 4} .\end{cases}
$$

Now, in the context of multiobjective, let us give the definitions of efficient or nondominated or Pareto optimal solutions for MOLTP. These are used instead of the optimal solution concept in a single objective TP.

Definition 3.1 (Pareto optimal solution for MOLTP). Let $S$ be the feasible region of (3.1). $\mathbf{x}^{*} \in S$ is said to be a Pareto optimal solution (strongly efficient or nondominated) if and only if there does not exist another $\mathbf{x} \in S$ such that $R\left(\tilde{f}^{k}(\mathbf{x})\right) \leq R\left(\tilde{f}^{k}\left(\mathbf{x}^{*}\right)\right)$ for all $k=1,2, \ldots, K$ and 


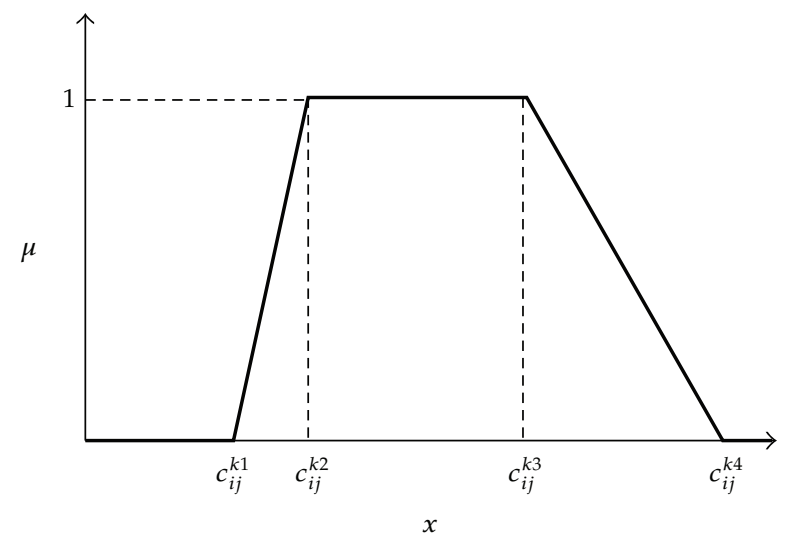

Figure 2: The membership function of the fuzzy cost coefficient $\tilde{c}_{i j}^{k}$.

$R\left(\tilde{f}^{k}(\mathbf{x})\right) \neq R\left(\tilde{f}^{k}\left(\mathbf{x}^{*}\right)\right)$ for at least one $k=1,2, \ldots, K$ where $R$ is the ranking function defined in (2.7).

Definition 3.2 (Compromise solution for MOLTP). A feasible solution $\mathbf{x}^{*} \in S$ is called a compromise solution of (3.1) if and only if $\mathbf{x}^{*} \in E$ and $R\left(\tilde{f}^{k}\left(\mathbf{x}^{*}\right)\right) \leq \wedge_{\mathbf{x} \in S} R(\tilde{f}(\mathbf{x}))$ where $R(\tilde{f}(\mathbf{x}))=\left(R\left(\tilde{f}^{1}(\mathbf{x})\right), R\left(\tilde{f}^{2}(\mathbf{x})\right), \ldots, R\left(\tilde{f}^{k}(\mathbf{x})\right)\right), \wedge$ stands for "min" operator and $E$ is the set of Pareto optimal solutions.

When the fuzzy cost coefficients are given as trapezoidal fuzzy number in the form $\tilde{c}_{i j}^{k}=\left(c_{i j}^{k 1}, c_{i j}^{k 2}, c_{i j}^{k 3}, c_{i j}^{k 4}\right)$ by means of (2.2) and (2.3), the objectives can be written as follows for each $k=1,2, \ldots, K$ :

$$
\begin{aligned}
\tilde{f}^{k}(\mathbf{x}) & =\sum_{i=1}^{m} \sum_{j=1}^{n}\left(c_{i j}^{k 1}, c_{i j}^{k 2}, c_{i j}^{k 3}, c_{i j}^{k 4}\right) x_{i j} \\
& =\left(\sum_{i=1}^{m} \sum_{j=1}^{n} c_{i j}^{k 1} x_{i j}, \sum_{i=1}^{m} \sum_{j=1}^{n} c_{i j}^{k 2} x_{i j}, \sum_{i=1}^{m} \sum_{j=1}^{n} c_{i j}^{k 3} x_{i j}, \sum_{i=1}^{m} \sum_{j=1}^{n} c_{i j}^{k 4} x_{i j}\right) .
\end{aligned}
$$

Since $\tilde{f}^{k}(\overline{\mathbf{x}})$ is a trapezoidal fuzzy number for a given $\overline{\mathbf{x}} \in S$, we need to define the minimum of fuzzy valued objective function. In this paper, the fuzziness in the objectives is handled with a fuzzy programming technique in the sense of multiobjective approach by means of (2.7). The ranking value of $\tilde{f}^{k}(\overline{\mathbf{x}})$ can be written as follows:

$$
R\left(\tilde{f}^{k}(\overline{\mathbf{x}})\right)=\frac{\sum_{i=1}^{m} \sum_{j=1}^{n} c_{i j}^{k 1} \bar{x}_{i j}+\sum_{i=1}^{m} \sum_{j=1}^{n} c_{i j}^{k 2} \bar{x}_{i j}+\sum_{i=1}^{m} \sum_{j=1}^{n} c_{i j}^{k 3} \bar{x}_{i j}+\sum_{i=1}^{m} \sum_{j=1}^{n} c_{i j}^{k 4} \bar{x}_{i j}}{4} .
$$

For obtaining a better ranking value, we want to find a feasible solution $\overline{\mathbf{x}} \in S$ that minimizes all of the characteristic points of the fuzzy objective value $\tilde{f}^{k}(\overline{\mathbf{x}})$, simultaneously. It implies 
that the less the characteristic points of fuzzy objective value the better (preferable) the solution. Thus, the minimum of objective function can be handled as

$$
\min \tilde{f}^{k}(\mathbf{x})=\left(\min \sum_{i=1}^{m} \sum_{j=1}^{n} c_{i j}^{k 1} x_{i j}, \min \sum_{i=1}^{m} \sum_{j=1}^{n} c_{i j}^{k 2} x_{i j}, \min \sum_{i=1}^{m} \sum_{j=1}^{n} c_{i j}^{k 3} x_{i j}, \min \sum_{i=1}^{m} \sum_{j=1}^{n} c_{i j}^{k 4} x_{i j}\right) .
$$

And with (3.6), (3.1) can be reformulated to the following problem in the sense of multiobjective approach [10]:

$$
\begin{aligned}
& \min f^{k 1}(\mathbf{x})=\sum_{i=1}^{m} \sum_{j=1}^{n} c_{i j}^{k 1} x_{i j}, \\
& \min f^{k 2}(\mathbf{x})=\sum_{i=1}^{m} \sum_{j=1}^{n} c_{i j}^{k 2} x_{i j}, \\
& \min f^{k 3}(\mathbf{x})=\sum_{i=1}^{m} \sum_{j=1}^{n} c_{i j}^{k 3} x_{i j}, \\
& \min f^{k 4}(\mathbf{x})=\sum_{i=1}^{m} \sum_{j=1}^{n} c_{i j}^{k 4} x_{i j},
\end{aligned}
$$

$$
\text { s.t. } \mathbf{x} \in S \text {. }
$$

Thus, by constructing four objectives for each $k \in K$, fuzziness in (3.1) is eliminated. In other words, our aim is to find the Pareto optimal solutions of (3.1) in the manner of multiobjective linear programming problems. We note that since $c_{i j}^{k 1} \leq c_{i j}^{k 2} \leq c_{i j}^{k 3} \leq c_{i j}^{k 4}$, the same order is valid between $f^{k 1}, f^{k 2}, f^{k 3}, f^{k 4}$ for each $k \in K$. So, we want to find a Pareto optimal solution that minimizes all of the objectives of (3.7a)-(3.7e) (all the characteristic points of all objectives). Certainly, the solution of (3.7a)-(3.7e) is not the same with the individual minima of objectives (3.7a)-(3.7d). In general, an optimal solution which simultaneously minimizes all objective functions in (3.7a)-(3.7e) does not always exist when the objective functions conflict with one another. When a certain Pareto optimal solution is selected, any improvement of one objective function can be achieved only at the expense of at least one of the other objective functions. A preferred compensatory compromise Pareto optimal solution is a solution which satisfies the decision maker's preferences and is preferred to all other solutions, taking into consideration all objectives contained in (3.7a)-(3.7e).

\section{Compensatory Operators}

There are several fuzzy aggregation operators. The detailed information about them exists in Zimmermann [11] and Tiryaki [14]. The most important aspect in the fuzzy approach is the compensatory or noncompensatory nature of the aggregation operator. Several investigators [11, 15-17] have discussed this aspect.

Using the linear membership function, Zimmermann [18] proposed the "min" operator model to the multiobjective linear problem (MOLP). It is usually used due to its easy computation. Although the "min" operator method has been proven to have several nice 
properties [16], the solution generated by min operator does not guarantee compensatory and Pareto optimality [19-21]. The biggest disadvantage of the aggregation operator "min" is that it is noncompensatory. In other words, the results obtained by the "min" operator represent the worst situation and cannot be compensated by other members which may be very good. On the other hand, the decision modeled with average operator is called fully compensatory in the sense that it maximizes the arithmetic mean value of all membership functions.

Zimmermann and Zysno [22] show that most of the decisions taken in the real world are neither noncompensatory (min operator) nor fully compensatory and suggested a class of hybrid compensatory operators with $\gamma$ compensation parameter.

Basing on the $\gamma$-operator, Werners [23] introduced the compensatory "fuzzy and" operator which is the convex combinations of min and arithmetical mean

$$
\mu_{\mathrm{and}}=\gamma \min _{i}\left(\mu_{i}\right)+\frac{(1-\gamma)}{m}\left(\sum_{i} \mu_{i}\right)
$$

where $0 \leq \mu_{i} \leq 1, i=1, \ldots, m$, and the magnitude of $\gamma \in[0,1]$ represent the grade of compensation.

Although this operator is not inductive and associative, this is commutative, idempotent, strictly monotonic increasing in each component, continuous, and compensatory. Obviously, when $\gamma=1$, this equation reduces to $\mu_{\text {and }}=\min$ (noncompensatory) operator. In literature, it is showed that the solution generated by Werners' compensatory "fuzzy and" operator does guarantee compensatory and Pareto optimality for MOLP [14, 16, 17, 2123]. Thus this operator is also suitable for our MOLTP. Therefore, due to its advantages, in this paper, we used Werners' compensatory "fuzzy and" operator to aggregate the multiple objectives.

\section{A Compensatory Approach to MOLTP with Fuzzy Cost Coefficients}

Now, we have a multi objective programming problem ((3.7a)-(3.7e)). In this paper, we used a fuzzy programming technique to solve this problem. So, we need to define the membership functions of objectives firstly.

\subsection{Constructing the Membership Functions of Objectives}

Now, the membership functions of $4 K$ objectives will be defined to apply our compensatory approach. Let $L_{k p}$ and $U_{k p}$ be the lower and upper bounds of the objective function $f^{k p}$ ( $k=$ $1,2, \ldots, K ; p=1,2,3,4)$, respectively. In the literature, there are two common ways of determining these bounds. The first way: solve the MOLTP as a single objective TP using each time only one objective and ignoring all others. Determine the corresponding values for every objective at each solution derived. And find the best $\left(L_{k p}\right)$ and the worst $\left(U_{k p}\right)$ values corresponding to the set of solutions. And the second way: by solving $8 \mathrm{~K}$ single-objective $\mathrm{TP}$, the lower and upper bounds $L_{k p}$ and $U_{k p}$ can also be determined for each objective $f^{k p}(\mathbf{x}), k=1,2, \ldots, K ; p=1,2,3,4$ as follows:

$$
L_{k p}=\min _{\mathbf{x} \in S} f^{k p}(\mathbf{x}), \quad U_{k p}=\max _{\mathbf{x} \in S} f^{k p}(\mathbf{x}),
$$


where $S$ is the feasible solution space, that is, satisfied supplydemand and non-negativity constraints. Here, we note that (5.1) will be used for determining the lower and upper bounds of objectives. There are several membership functions in the literature, for example, linear, hyperbolic and piecewise linear, and so forth. For simplicity, in this paper, we used a linear membership function

$$
\mu_{k p}\left(f^{k p}\right)= \begin{cases}1, & f^{k p}<L_{k p} \\ \frac{U_{k p}-f^{k p}}{U_{k p}-L_{k p}}, & L_{k p} \leq f^{k p} \leq U_{k p} \\ 0, & f^{k p}>U_{k p} .\end{cases}
$$

Here, $L_{k} \neq U_{k}, k=1,2, \ldots, K$ and in the case of $L_{k}=U_{k}, \mu_{k p}\left(f^{k p}(\mathbf{x})\right)=1$. The membership function $\mu_{k p}\left(f^{k p}\right)$ is linear and strictly monotone decreasing for $f^{k p}$ in the interval $\left[L_{k p}, U_{k p}\right]$.

Using Zimmermann's minimum operator [18], MOLTP can be written as

$$
\begin{gathered}
\max _{x} \min _{k p} \mu_{k p}\left(f^{k p}(\mathbf{x})\right), \\
\text { s.t. } \quad \mathbf{x} \in S .
\end{gathered}
$$

By introducing an auxiliary variable $\lambda$,

$$
\min _{k p} \mu_{k p}\left(f^{k p}(\mathbf{x})\right)=\lambda \Longrightarrow \mu_{k p}\left(f^{k p}(\mathbf{x})\right) \geq \lambda
$$

(5.3) can be transformed into the following equivalent conventional LP problem:

$$
\begin{array}{ll}
\max \lambda, & \\
\text { s.t. } & \mu_{k p}\left(f^{k p}(\mathbf{x})\right) \geq \lambda, \quad k=1, \ldots, K ; p=1,2,3,4, \\
& \mathbf{x} \in S \\
& \lambda \in[0,1] .
\end{array}
$$

Here, we note that (5.5) is the "min" operator model for MOLTP. Its optimal objective value denotes the maximizing value of the least satisfaction level among all objectives. And it can also be interpreted as the "most basic satisfaction" that each objective in the transportation system can attain.

\subsection{Werners' Compensatory $\mu_{\text {and }}$ Operator for MOLTP with Fuzzy Cost Coefficients}

It is pointed out that Zimmermann's min operator model is a noncompensatory model and does not always yield a Pareto optimal solution [19-21]. So we used Werners' $\mu_{\text {and }}$ operator to aggregate our objectives' membership functions. 
For every objective, after satisfying its most basic satisfaction level in the transportation system, to promote its satisfaction degree as high as possible, we can make the following arrangement:

$$
\mu_{k p}\left(f^{k p}(\mathbf{x})\right)=\lambda+\lambda_{k p}
$$

where $\lambda=\min \mu_{k p}\left(\tilde{f}_{k p}\right)$.

This arrangement is introduced to the constraints with the following expressions:

(i) $\mu_{k p}\left(f^{k p}(\mathbf{x})\right)=\lambda+\lambda_{k p}$,

(ii) $\lambda+\lambda_{k p} \leq 1$.

So, $\mu_{\text {and }}$ used in this paper (4.1) can be formed as

$$
\begin{aligned}
& \mu_{\text {and }}=\gamma \min _{k p}\left(\mu_{k p}\right)+\frac{(1-\gamma)}{4 K}\left(\mu_{11}+\mu_{12}+\mu_{13}+\mu_{14}+\mu_{21}+\cdots+\mu_{K 4}\right), \\
& \mu_{\text {and }}=\gamma \lambda+\frac{(1-\gamma)}{4 K}\left(\left(\lambda+\lambda_{11}\right)+\left(\lambda+\lambda_{12}\right)+\left(\lambda+\lambda_{13}\right)+\left(\lambda+\lambda_{14}\right)+\left(\lambda+\lambda_{21}\right) \cdots+\left(\lambda+\lambda_{K 4}\right)\right), \\
& \mu_{\text {and }}=\lambda+\frac{(1-\gamma)}{4 K}\left(\lambda_{11}+\lambda_{12}+\lambda_{13}+\lambda_{14}+\lambda_{21}+\cdots+\lambda_{K 4}\right)
\end{aligned}
$$

where the magnitude of $\gamma \in[0,1]$ represents the grade of compensation. Obviously, when $\gamma=1$ and $0, \mu_{\text {and }}=$ " $\min$ " operator and $\mu_{\text {and }}=$ "average" operator, respectively.

Therefore, depending on the compensation parameter $\gamma,(5.5)$ is converted to the following compensatory model that is solved for obtaining compromise Pareto optimal solutions for MOLTP:

$$
\begin{array}{ll}
\max & \mu_{\mathrm{and}}=\lambda+\frac{(1-\gamma)}{4 K}\left[\sum_{k=1}^{K} \sum_{p=1}^{4} \lambda_{k p}\right], \\
\text { s.t.: } \quad \mathbf{x} \in S & \\
& \mu_{k p}\left(f^{k p}(\mathbf{x})\right) \geq \lambda+\lambda_{k p}, \quad \forall k=1,2, \ldots, K ; \forall p=1,2,3,4, \\
& \lambda+\lambda_{k p} \leq 1, \quad \forall k=1,2, \ldots, K ; \forall p=1,2,3,4, \\
& \gamma, \lambda, \lambda_{k p} \in[0,1], \\
& x_{i j} \geq 0, \quad i=1,2, \ldots, m ; j=1,2, \ldots, n .
\end{array}
$$

It is noted that in order to avoid some possible computational errors in solution process, we added the condition (i) as $\mu_{k p} \geq \lambda+\lambda_{k p}$ to the formulated problem (5.8). So, our compensatory model generates compensatory compromise Pareto optimal solutions for MOLTP.

We shall give this assertion in the following theorem. 
Table 1: The penalties of the single objective in Example 6.1.

\begin{tabular}{cccc}
\hline & 1 & 2 & 3 \\
\hline 1 & $(1,2,10,100)$ & $(2,4,20,80)$ & $(3,6,20,60)$ \\
2 & $(4,8,10,40)$ & $(5,10,20,30)$ & $(6,7,7,10)$ \\
\hline
\end{tabular}

Table 2: Bound values of objective.

\begin{tabular}{lcccc}
\hline & $f_{11}$ & $f_{12}$ & $f_{13}$ & $f_{14}$ \\
\hline$L_{k p}$ & 540 & 730 & 1590 & 6400 \\
$U_{k p}$ & 540 & 1030 & 2370 & 6700 \\
\hline
\end{tabular}

Theorem 5.1. If $\left(\mathbf{x}, \boldsymbol{\lambda}^{\mathbf{x}}\right)$ is an optimal solution of problem (5.8), then $\mathbf{x}$ is a Pareto optimal solution for MOLTP, where $\lambda^{\mathrm{x}}=\left(\lambda^{\mathrm{x}}, \lambda_{11}^{\mathrm{x}}, \lambda_{12}^{\mathrm{x}}, \lambda_{13}^{\mathrm{x}}, \lambda_{14}^{\mathrm{x}}, \ldots, \lambda_{K 1}^{\mathrm{x}}, \lambda_{K 2}^{\mathrm{x}}, \lambda_{K 3}^{\mathrm{x}}, \lambda_{K 4}^{\mathrm{x}}\right)$.

If required, the proof of the theorem can be found in [23]. Also, Pareto optimality test [24] can be applied to the solutions of (5.8), and it could be seen that these solutions are Pareto optimal for MOLTP.

We also note here that our approach is valid for single objective TPs with fuzzy cost coefficients since our compensatory approach could be applied to four objectives which are constructed from the original single objective function of TP.

\section{Illustrative Examples}

In this section, two numerical examples are given to explain our approach. The first example contains only one objective. Second one dealt with the multiobjective version.

\subsection{A Single Objective Transportation Problem with Fuzzy Cost Coefficients}

In this paper, we studied MOLTP with crisp supply\&demand parameters but fuzzy costs which are given as trapezoidal fuzzy numbers. To our knowledge this combination is not studied in the literature. But [8] dealt with the single objective version of TP which is studied in our paper. In [8], single objective TP is converted to a bicriterial TP, and an algorithm is given to solve this bicriterial problem by means of parametric programming. The numerical example that is given in [8] is as follows:

Supplies: $a_{1}=70 ; a_{2}=70$;

Demands: $b_{1}=30 ; b_{2}=30 ; b_{3}=80$;

Penalties of objective: $c_{i j}^{1}$ see Table 1 .

Using (5.1), the lower and upper bounds of objectives are determined to construct the membership functions as mentioned in Table 2. 
Using (5.8), the compensatory model is constructed as follows:

$$
\begin{array}{ll}
\max & \mu_{\mathrm{and}}=\lambda+\frac{(1-\gamma)}{4}\left[\sum_{p=1}^{4} \lambda_{1 p}\right] \\
\text { s.t. } \quad & \sum_{j=1}^{3} x_{1 j}=70, \quad \sum_{j=1}^{3} x_{2 j}=70, \\
& \sum_{i=1}^{2} x_{i 1}=30, \quad \sum_{i=1}^{2} x_{i 2}=30, \quad \sum_{i=1}^{2} x_{i 3}=80, \\
& f^{11}(\mathbf{x})+0 \cdot\left(\lambda+\lambda_{11}\right) \leq 540, \quad f^{12}(\mathbf{x})+300\left(\lambda+\lambda_{12}\right) \leq 1030, \\
& f^{13}(\mathbf{x})+780\left(\lambda+\lambda_{13}\right) \leq 2370, \quad f^{14}(\mathbf{x})+300\left(\lambda+\lambda_{14}\right) \leq 6700, \\
& \lambda+\lambda_{1 p} \leq 1, \quad p=1,2,3,4, \\
& \lambda, \lambda_{1 p}, \gamma \in[0,1], \quad p=1,2,3,4 \\
& x_{i j} \geq 0 \quad(i=1,2 ; \quad j=1,2,3) .
\end{array}
$$

This compensatory model generates the following compensatory compromise Pareto optimal solutions for different 11 values of the compensation parameter $\gamma$ with 0.1 increment.

For the value of $\gamma=0$, we obtained three alternative solutions

$$
\begin{gathered}
\mathbf{X}^{1 *}=\left\{\begin{array}{c}
x_{11}=30, x_{12}=30, x_{13}=10 \\
x_{21}=0, x_{22}=0, x_{23}=70
\end{array}\right\}, \\
f^{11}\left(\mathbf{X}^{1 *}\right)=540, \quad f^{12}\left(\mathbf{X}^{1 *}\right)=730, \quad f^{13}\left(\mathbf{X}^{1 *}\right)=1590, \quad f^{14}\left(\mathbf{X}^{1 *}\right)=6700 ; \\
\mathbf{X}^{2 *}=\left\{\begin{array}{l}
x_{11}=0, x_{12}=30, x_{13}=40 \\
x_{21}=30, x_{22}=0, x_{23}=40
\end{array}\right\}, \\
f^{11}\left(\mathbf{X}^{2 *}\right)=540, \quad f^{12}\left(\mathbf{X}^{2 *}\right)=880, \quad f^{13}\left(\mathbf{X}^{2 *}\right)=1980, \quad f^{14}\left(\mathbf{X}^{2 *}\right)=6400 ; \\
\mathbf{X}^{3 *}=\left\{\begin{array}{c}
x_{11}=10, x_{12}=30, x_{13}=30 \\
x_{21}=20, x_{22}=0, x_{23}=50
\end{array}\right\}, \\
f^{11}\left(\mathbf{X}^{3 *}\right)=540, \quad f^{12}\left(\mathbf{X}^{3 *}\right)=830, \quad f^{13}\left(\mathbf{X}^{3 *}\right)=1850, \quad f^{14}\left(\mathbf{X}^{3 *}\right)=6500 .
\end{gathered}
$$

The solution $\mathbf{X}^{3 *}$ is obtained for the value of $\gamma=0.1-\gamma=1.0$. 
Table 3: The penalties of the first objective in Example 6.2.

\begin{tabular}{ccccc}
\hline & 1 & 2 & 3 & 4 \\
\hline 1 & $(6,12,15,20)$ & $(7,9,12,16)$ & $(6,9,10,12)$ & $(3,7,8,16)$ \\
2 & $(15,16,17,20)$ & $(13,16,18,19)$ & $(18,20,21,25)$ & $(10,12,14,16)$ \\
3 & $(6,9,14,20)$ & $(10,12,15,20)$ & $(6,9,10,12)$ & $(15,20,21,24)$ \\
\hline
\end{tabular}

These solutions imply following fuzzy objective values:

$$
\begin{aligned}
& \tilde{f}^{1}\left(\mathbf{X}^{1 *}\right)=(540,730,1590,6700), \\
& \tilde{f}^{1}\left(\mathbf{X}^{2 *}\right)=(540,880,1980,6400), \\
& \tilde{f}^{1}\left(\mathbf{X}^{3 *}\right)=(540,830,1850,6500) .
\end{aligned}
$$

In [8], three alternative solution sets are obtained. Two of them are the same as our solution sets $\mathbf{X}^{1 *}, \mathbf{X}^{2 *}$. And the other solution set is

$$
\begin{gathered}
\mathbf{X}^{4 *}=\left\{\begin{array}{c}
x_{11}=0, x_{12}=0, x_{13}=70 \\
x_{21}=30, x_{22}=30, x_{23}=10
\end{array}\right\}, \\
f^{11}\left(\mathbf{X}^{4 *}\right)=540, \quad f^{12}\left(\mathbf{X}^{4 *}\right)=1030, \quad f^{13}\left(\mathbf{X}^{3 *}\right)=2370, \quad f^{14}\left(\mathbf{X}^{3 *}\right)=6400,
\end{gathered}
$$

with the fuzzy objective value $\tilde{f}^{1}\left(\mathbf{X}^{4 *}\right)=(540,1030,2370,6400)$.

To compare our results, the ranking function which is defined in (2.7) can be used. The ranking values of the solutions are as follows:

$$
\begin{array}{ll}
R\left(\tilde{f}^{1}\left(\mathbf{X}^{1 *}\right)\right)=2390, & R\left(\tilde{f}^{1}\left(\mathbf{X}^{2 *}\right)\right)=2450, \\
R\left(\tilde{f}^{1}\left(\mathbf{X}^{3 *}\right)\right)=2430, & R\left(\tilde{f}^{1}\left(\mathbf{X}^{4 *}\right)\right)=2585 .
\end{array}
$$

As it can be seen from the ranking values, our compensatory model generates better fuzzy objective values according to the ranking function (2.7).

\subsection{Multiobjective Transportation Problem with Fuzzy Cost Coefficients}

Let us consider a MOLTP with the following characteristics:

supplies: $a_{1}=24 ; a_{2}=8 ; a_{3}=18$;

demands: $b_{1}=11 ; b_{2}=9 ; b_{3}=21 ; b_{4}=9$;

penalties of the first objective: $c_{i j}^{1}$ see Table 3 ;

penalties of the second objective: $c_{i j}^{2}$ see Table 4 . 
Table 4: The penalties of the second objective in Example 6.2.

\begin{tabular}{ccccc}
\hline & 1 & 2 & 3 & 4 \\
\hline 1 & $(2,3,6,10)$ & $(4,8,10,14)$ & $(8,10,11,12)$ & $(5,8,10,12)$ \\
2 & $(6,11,13,20)$ & $(10,11,12,14)$ & $(16,18,20,25)$ & $(14,16,17,20)$ \\
3 & $(3,4,5,8)$ & $(8,10,11,14)$ & $(7,10,10,12)$ & $(14,15,15,18)$ \\
\hline
\end{tabular}

Table 5: Bound values of objectives.

\begin{tabular}{lllllllll}
\hline & $f_{11}$ & $f_{12}$ & $f_{13}$ & $f_{14}$ & $f_{21}$ & $f_{22}$ & $f_{23}$ & $f_{24}$ \\
\hline$L_{k p}$ & 330 & 472 & 568 & 760 & 285 & 411 & 475 & 574 \\
$U_{k p}$ & 513 & 697 & 787 & 972 & 452 & 532 & 603 & 754 \\
\hline
\end{tabular}

Using (5.1), the lower and upper bounds of the objectives are determined to construct the membership functions as mentioned in Table 5.

Using (5.8), the compensatory model is constructed as follows:

$$
\begin{array}{ll}
\max \mu_{\text {and }}=\lambda+\frac{(1-\gamma)}{8}\left[\sum_{k=1}^{2} \sum_{p=1}^{4} \lambda_{k p}\right], & \\
\text { s.t. } \quad \sum_{j=1}^{4} x_{1 j}=24, \quad \sum_{j=1}^{4} x_{2 j}=8, \quad \sum_{j=1}^{4} x_{3 j}=18, \\
\sum_{i=1}^{3} x_{i 1}=11, \quad \sum_{i=1}^{3} x_{i 2}=9, \quad \sum_{i=1}^{3} x_{i 3}=21, \quad \sum_{i=1}^{3} x_{i 4}=9, \\
f^{11}(\mathbf{x})+183\left(\lambda+\lambda_{11}\right) \leq 513, \quad f^{12}(\mathbf{x})+225\left(\lambda+\lambda_{12}\right) \leq 697, \\
f^{13}(\mathbf{x})+219\left(\lambda+\lambda_{13}\right) \leq 787, \quad f^{14}(\mathbf{x})+212\left(\lambda+\lambda_{14}\right) \leq 972, \\
f^{21}(\mathbf{x})+167\left(\lambda+\lambda_{21}\right) \leq 452, \quad f^{22}(\mathbf{x})+121\left(\lambda+\lambda_{22}\right) \leq 532, \\
f^{23}(\mathbf{x})+128\left(\lambda+\lambda_{23}\right) \leq 603, \quad f^{24}(\mathbf{x})+180\left(\lambda+\lambda_{24}\right) \leq 754, \\
\lambda+\lambda_{k p} \leq 1, \quad \forall k=1,2 ; p=1,2,3,4, \\
\lambda, \lambda_{k p}, \gamma \in[0,1], \quad \forall k=1,2 ; p=1,2,3,4, \\
x_{i j} \geq 0 \quad(i=1,2,3 ; j=1,2,3,4) .
\end{array}
$$

By solving (6.6), the results for different 11 values of the compensation parameter $\gamma$ with 0.1 increment are obtained and given in Table 6 . The results are the values of objective functions $f^{k p}(k=1,2 ; p=1,2,3,4)$; the satisfactory levels of the objectives corresponding to solution $\mathbf{x}$, (i.e. the values of membership functions) $\mu_{k p}(k=1,2 ; p=1,2,3,4)$; the most basic satisfactory level $\lambda$; the compensation satisfactory level $\mu_{\text {and }}$, respectively.

So, our compensatory model generates the following compensatory compromise Pareto optimal solutions $\mathbf{X}^{1 *}, \mathbf{X}^{2 *}$, and $\mathbf{X}^{3 *}$ for our MOLTP. 


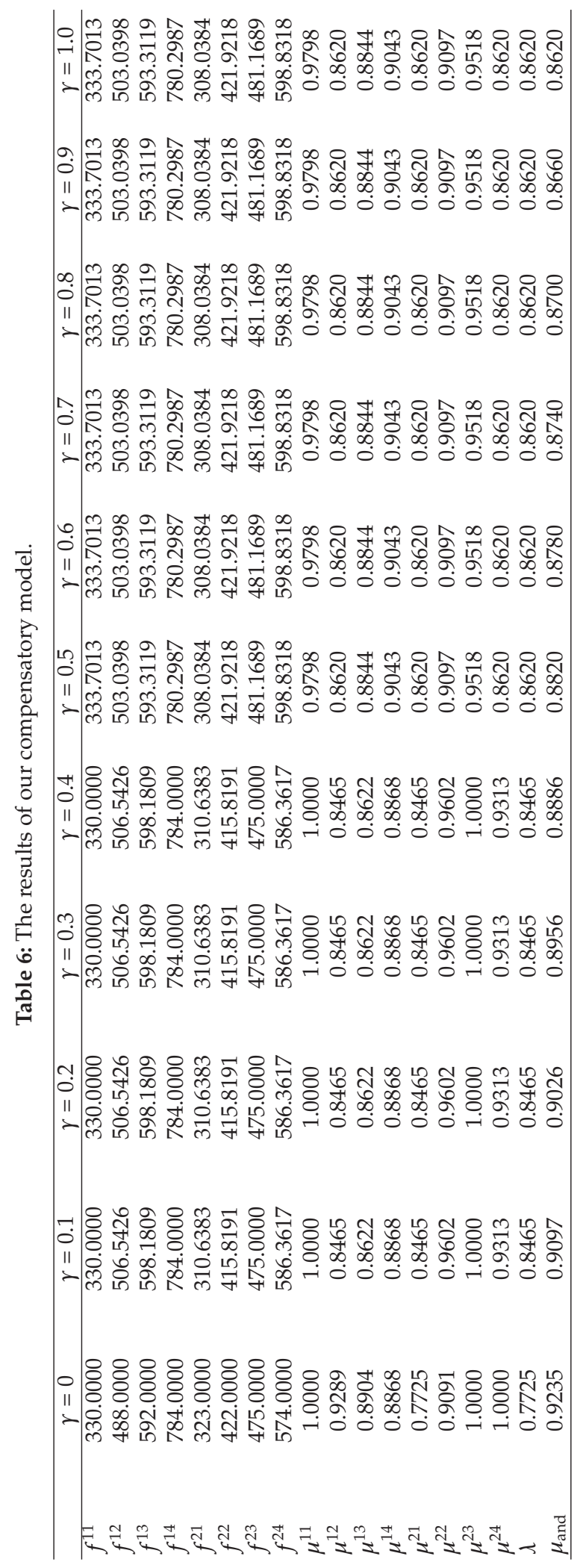


For the value of $\gamma=0$,

$$
\mathbf{X}^{1 *}=\left\{\begin{array}{c}
x_{11}=0, x_{12}=1, x_{13}=14, x_{14}=9 \\
x_{21}=0, x_{22}=8, x_{23}=0, x_{24}=0 \\
x_{31}=11, x_{32}=0, x_{33}=7, x_{34}=0
\end{array}\right\},
$$

$f^{11}\left(\mathbf{X}^{1 *}\right)=330, \quad f^{12}\left(\mathbf{X}^{1 *}\right)=488, \quad f^{13}\left(\mathbf{X}^{1 *}\right)=592, \quad f^{14}\left(\mathbf{X}^{1 *}\right)=784$,

$f^{21}\left(\mathbf{X}^{1 *}\right)=323, \quad f^{22}\left(\mathbf{X}^{1 *}\right)=422, \quad f^{23}\left(\mathbf{X}^{1 *}\right)=475, \quad f^{24}\left(\mathbf{X}^{1 *}\right)=574$.

For the value of $\gamma=0.1-\gamma=0.4$,

$$
\begin{gathered}
\mathbf{X}^{2 *}=\left\{\begin{array}{c}
x_{11}=6.1809, x_{12}=1, x_{13}=7.8191, x_{14}=9 \\
x_{21}=0, x_{22}=8, x_{23}=0, x_{24}=0 \\
x_{31}=4.8191, x_{32}=0, x_{33}=13.1809, x_{34}=0
\end{array}\right\}, \\
f^{11}\left(\mathbf{X}^{2 *}\right)=330, \quad f^{12}\left(\mathbf{X}^{2 *}\right)=506.5426, \\
f^{13}\left(\mathbf{X}^{2 *}\right)=598.1809, \quad f^{14}\left(\mathbf{X}^{2 *}\right)=784 \\
f^{21}\left(\mathbf{X}^{2 *}\right)=310.6383, \quad f^{22}\left(\mathbf{X}^{2 *}\right)=415.8191, \\
f^{23}\left(\mathbf{X}^{2 *}\right)=475, \quad f^{24}\left(\mathbf{X}^{2 *}\right)=586.3617 .
\end{gathered}
$$

For the value of $\gamma=0.5-\gamma=1.0$,

$$
\begin{gathered}
\mathbf{X}^{3 *}=\left\{\begin{array}{r}
x_{11}=5.0133, x_{12}=2.2338, x_{13}=7.7530, x_{14}=9 \\
x_{21}=1.2338, x_{22}=6.7662, x_{23}=0, x_{24}=0 \\
x_{31}=4.7530, x_{32}=0, x_{33}=13.2470, x_{34}=0
\end{array}\right\}, \\
f^{11}\left(\mathbf{X}^{3 *}\right)=333.7013, \quad f^{12}\left(\mathbf{X}^{3 *}\right)=503.0398, \\
f^{13}\left(\mathbf{X}^{3 *}\right)=593.3119, \quad f^{14}\left(\mathbf{X}^{3 *}\right)=780.2987, \\
f^{21}\left(\mathbf{X}^{3 *}\right)=308.0334, \quad f^{22}\left(\mathbf{X}^{3 *}\right)=421.9218 \\
f^{23}\left(\mathbf{X}^{3 *}\right)=481.1689, \quad f^{24}\left(\mathbf{X}^{3 *}\right)=598.8318 .
\end{gathered}
$$


These solutions imply following fuzzy objective values for our MOLTP:

$$
\begin{aligned}
& \tilde{f}^{1}\left(\mathbf{X}^{1 *}\right)=(488,592,158,192) \\
& \tilde{f}^{1}\left(\mathbf{X}^{2 *}\right)=(506.5426,598.1809,176.5426,185.8191) \\
& \tilde{f}^{1}\left(\mathbf{X}^{3 *}\right)=(503.0398,593.3119,169.3385,186.9868) \\
& \tilde{f}^{2}\left(\mathbf{X}^{1 *}\right)=(422,475,99,99) \\
& \tilde{f}^{2}\left(\mathbf{X}^{2 *}\right)=(415.8191,475,105.1808,111.3617) \\
& \tilde{f}^{2}\left(\mathbf{X}^{3 *}\right)=(421.9218,481.1689,113.8834,117.6629) .
\end{aligned}
$$

All of these solutions pointed out that for all possible values of $\tilde{c}_{i j}^{k}(i=1,2,3 ; j=$ $1,2,3,4 ; k=1,2)$, the certainly transported amounts are

$$
\left\{\begin{array}{c}
x_{14}=9, x_{23}=0 \\
x_{24}=0, x_{32}=0, \\
x_{34}=0
\end{array}\right\} .
$$

And also, the least transported amounts are

$$
\left\{\begin{array}{c}
x_{12} \geq 1, x_{13} \geq 7.7530 \\
x_{22} \geq 6.7662 \\
x_{31} \geq 4.7530, x_{33} \geq 7
\end{array}\right\}
$$

For $\gamma=1, \mu_{\text {and }}$ equals to $\min$ (noncompensatory) operator that is $\mu_{\text {and }}=\min _{k p} \mu_{f_{k p}}=$ 0.8620 and gives the solution $X^{3 *}$. This solution remains the same for $\gamma=[0.5,1]$.

For $\gamma=0, \mu_{\text {and }}$ equals to average operator (full compensatory) operator, that is, $\mu_{\text {and }}=$ $\min (1 / 4 K) \sum \mu_{f_{k p}}=0.9235$ and gives the solution $\mathbf{X}^{1 *}$.

These solutions and the values of all membership functions are offered to decision maker $(\mathrm{DM})$. If $\mathrm{DM}$ is not satisfied with the proposed solution then he/she could assign the weights $w_{k},\left(w_{k}>0, \sum_{k=1}^{K} w_{k}=1\right)$ on his/her objectives $f^{k}, k=1,2$. In this case, the weights $w_{k}$ are inserted to the compensatory model as the following manner:

$$
\begin{gathered}
\frac{\mu_{k p}\left(f^{k p}\right)}{w_{k}} \geq \lambda+\lambda_{k p}, \quad \forall k=1,2 ; p=1,2,3,4, \\
w_{k}\left(\lambda+\lambda_{k p}\right) \leq 1, \quad \forall k=1,2 ; p=1,2,3,4,
\end{gathered}
$$

instead of the constraints

$$
\mu_{k p}\left(f^{k p}(\mathbf{x})\right) \geq \lambda+\lambda_{k p}, \quad \forall k=1,2 ; p=1,2,3,4
$$




\section{Conclusions}

MOLTP which is a well-known problem in the literature has wide practical applications in manpower planning, personnel allocation, inventory control, production planning, and so forth. In this paper, we deal with MOLTP whose costs coefficients are given as trapezoidal fuzzy numbers. We assume that the supply and demand quantities are precisely known. The fuzziness in the objectives is handled with a fuzzy programming technique in the sense of multiobjective approach. And a compensatory approach is given by using Werner's $\mu_{\text {and }}$ operator. Our approach generates compromise solutions which are both compensatory and Pareto optimal for MOLTP. It is known that Zimmerman's "min" operator is not compensatory and also does not guarantee to generate the Pareto optimal solutions. Werner's $\mu_{\text {and }}$ operator is useful about computational efficiency and always generates Pareto optimal solutions. The proposed approach also makes it possible to overcome the nonlinear nature owing to the fuzziness in the costs.

This paper discussed MOLTP with fuzzy cost coefficient. For further work, MOLTP with fuzzy supply\&demand quantities and also multi-index form of this problem could be considered.

\section{References}

[1] M. L. Hussein, "Complete solutions of multiple objective transportation problems with possibilistic coefficients," Fuzzy Sets and Systems, vol. 93, no. 3, pp. 293-299, 1998.

[2] S. K. Das, A. Goswami, and S. S. Alam, "Multiobjective transportation problem with interval cost, source and destination parameters," European Journal of Operational Research, vol. 117, no. 1, pp. 100$112,1999$.

[3] M. Ahlatcioglu, M. Sivri, and N. ve Guzel, "Transportation of the fuzzy amounts using the fuzzy cost," Journal of Marmara for Pure and Applied Sciences, vol. 18, no. 2, pp. 139-155, 2004.

[4] S.-T. Liu and C. Kao, "Solving fuzzy transportation problems based on extension principle," European Journal of Operational Research, vol. 153, no. 3, pp. 661-674, 2004.

[5] J. Chiang, "The optimal solution of the transportation problem with fuzzy demand and fuzzy product," Journal of Information Science and Engineering, vol. 21, no. 2, pp. 439-451, 2005.

[6] E. E. Ammar and E. A. Youness, "Study on multiobjective transportation problem with fuzzy numbers," Applied Mathematics and Computation, vol. 166, no. 2, pp. 241-253, 2005.

[7] S. Islam and T. K. Roy, "A new fuzzy multi-objective programming: entropy based geometric programming and its application of transportation problems," European Journal of Operational Research, vol. 173, no. 2, pp. 387-404, 2006.

[8] S. Chanas and D. Kuchta, "A concept of the optimal solution of the transportation problem with fuzzy cost coefficients," Fuzzy Sets and Systems, vol. 82, no. 3, pp. 299-305, 1996.

[9] S. Pramanik and T. K. Roy, "Multiobjective transportation model with Fuzzy parameters: priority based Fuzzy goal programming approach," Journal of Transportation Systems Engineering and Information Technology, vol. 8, no. 3, pp. 40-48, 2008.

[10] S. Okada and T. Soper, "A shortest path problem on a network with fuzzy arc lengths," Fuzzy Sets and Systems, vol. 109, no. 1, pp. 129-140, 2000.

[11] H.-J. Zimmermann, Fuzzy Set Theory-and Its Applications, Kluwer Academic Publishers, Boston, Mass, USA, 4th edition, 2001.

[12] Y.-J. Lai and C. L. Hwang, Fuzzy Mathematical Programming, vol. 394 of Lecture Notes in Economics and Mathematical Systems, Springer, Berlin, Germany, 1992.

[13] R. R. Yager, "A procedure for ordering fuzzy subsets of the unit interval," Information Sciences, vol. 24, no. 2, pp. 143-161, 1981.

[14] F. Tiryaki, "Interactive compensatory fuzzy programming for decentralized multi-level linear programming (DMLLP) problems," Fuzzy Sets and Systems, vol. 157, no. 23, pp. 3072-3090, 2006.

[15] Y.-J. Lai and C. L. Hwang, Fuzzy Multiple Objective Decision Making, vol. 404 of Lecture Notes in Economics and Mathematical Systems, Springer, Berlin, Germany, 1994. 
[16] M. K. Luhandjula, "Compensatory operators in fuzzy linear programming with multiple objectives," Fuzzy Sets and Systems, vol. 8, no. 3, pp. 245-252, 1982.

[17] H.-S. Shih and E. Stanley Lee, "Compensatory fuzzy multiple level decision making," Fuzzy Sets and Systems, vol. 114, no. 1, pp. 71-87, 2000.

[18] H.-J. Zimmermann, "Fuzzy programming and linear programming with several objective functions," Fuzzy Sets and Systems, vol. 1, no. 1, pp. 45-55, 1978.

[19] S.-Mi. Guu and Y.-K. Wu, "Weighted coefficients in two-phase approach for solving the multiple objective programming problems," Fuzzy Sets and Systems, vol. 85, no. 1, pp. 45-48, 1997.

[20] E. S. Lee and R.-J. Li, "Fuzzy multiple objective programming and compromise programming with Pareto optimum," Fuzzy Sets and Systems, vol. 53, no. 3, pp. 275-288, 1993.

[21] Y. K. Wu and S. M. Guu, "A compromise model for solving fuzzy multiple objective problems," Journal of the Chinese Institute of Industrial Engineers, vol. 18, no. 5, pp. 87-93, 2001.

[22] H.-J. Zimmermann and P. Zysno, "Latent connectives in human decision making," Fuzzy Sets and Systems, vol. 4, no. 1, pp. 37-51, 1980.

[23] B. M. Werners, "Aggregation models in mathematical programming," in Mathematical Models for Decision Support (Val d'Isère, 1987), vol. 48, pp. 295-305, Springer, Berlin, Germany, 1988.

[24] M. Ahlatcioglu and F. Tiryaki, "Interactive fuzzy programming for decentralized two-level linear fractional programming (DTLLFP) problems," Omega, vol. 35, no. 4, pp. 432-450, 2007. 


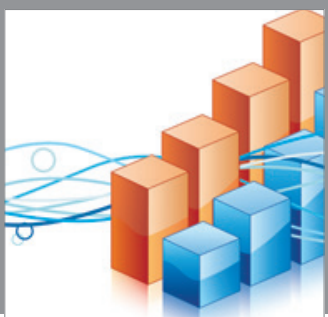

Advances in

Operations Research

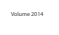

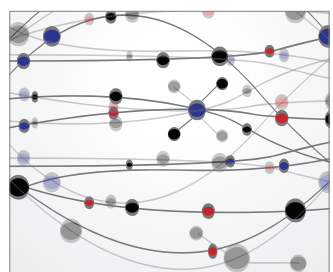

\section{The Scientific} World Journal
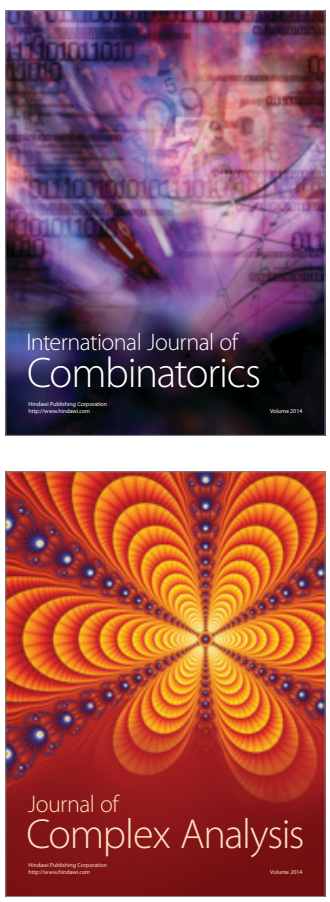

International Journal of

Mathematics and

Mathematical

Sciences
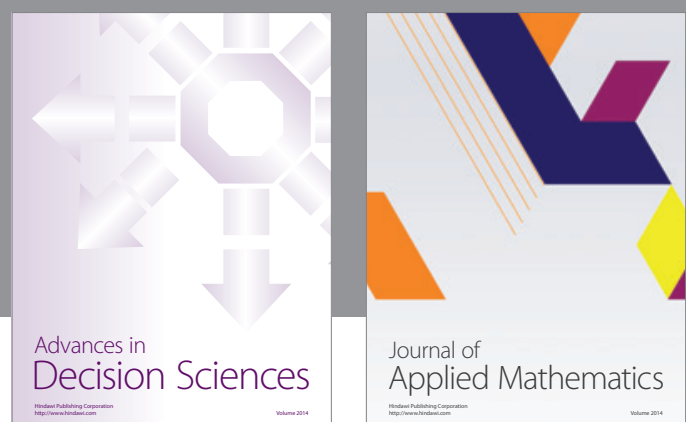

Journal of

Applied Mathematics
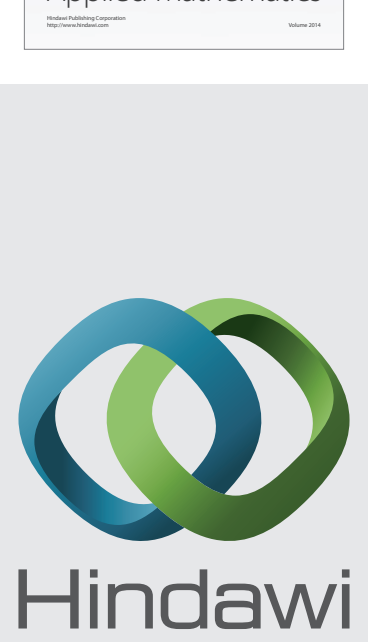

Submit your manuscripts at http://www.hindawi.com
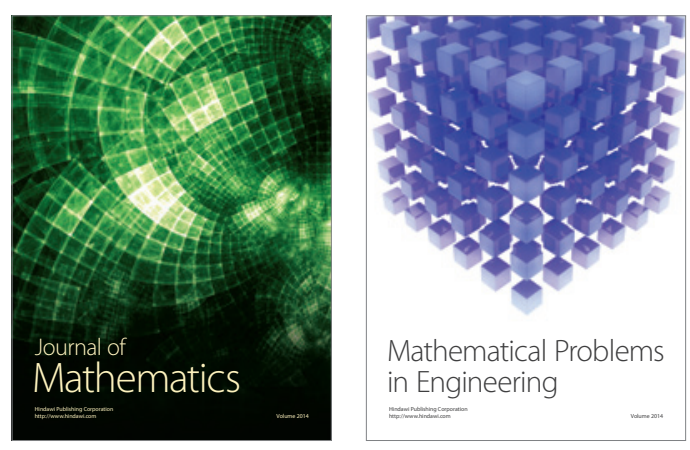

Mathematical Problems in Engineering
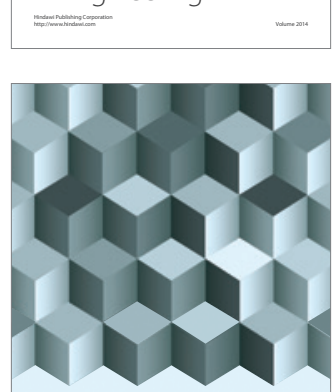

Journal of

Function Spaces
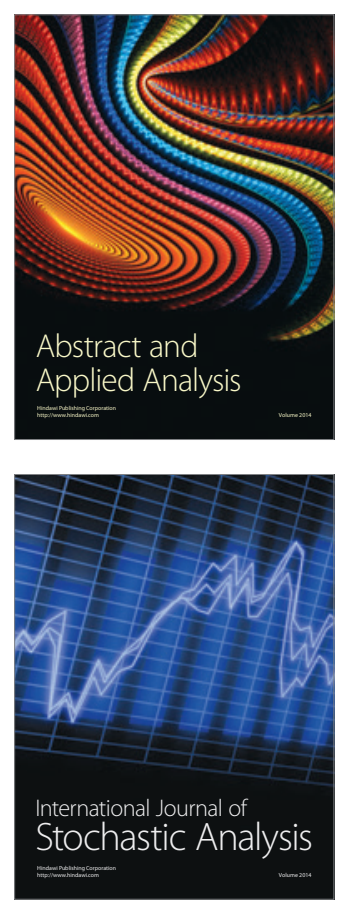

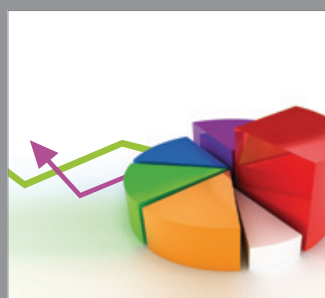

ournal of

Probability and Statistics

Promensencen
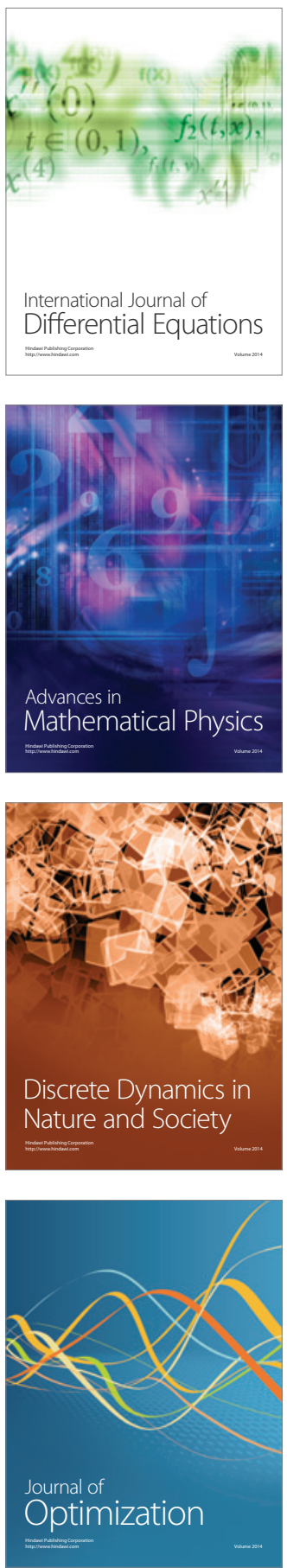